\title{
Effect of methyl methacrylate grafted kenaf on mechanical properties of polyvinyl chloride/ethylene vinyl acetate composites
}

\begin{abstract}
In this work, the effect of grafted and ungrafted kenaf fiber on tensile, flexural, impact and hardness properties of polyvinyl chloride/ethylene vinyl acetate composites were studied. Grafted fibers were obtained from graft copolymerization reaction where methyl methacrylate was used as a monomer. The fiber loadings were varied from $0 \%$ to $30 \%$. The addition of kenaf fiber had caused a decline in the impact and tensile strength of the PVC/EVA composite. Results revealed that the presence of PMMA on the surface of kenaf fiber has further enhanced the efficiency of stress transfer from the matrix to the fiber grafting thus improved the interfacial adhesion between the fiber and PVC/EVA matrix.
\end{abstract}

Keyword: Polymer-matrix composites; Fiber/matrix bond; Mechanical properties; Surface treatment 